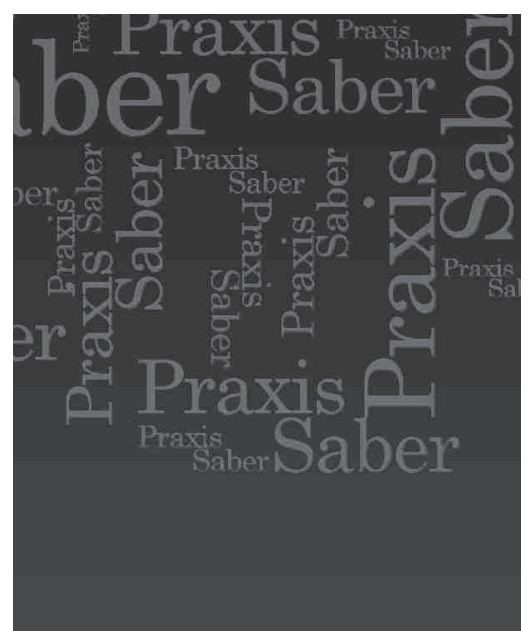

Graciela Concepción Messina Candidata a Doctora

DIE-CINVESTAV

Ciudad de México

Consultora de organismos

nacionales e internacionales

messinagra@prodigy.net.mx

Artículo de Reflexión

Recibido: 11 de Septiembre de 2011 Aprobado: 30 de Noviembre de 2011



Revista de Investigación y Pedagogía Maestría en Educación. Uptc

\section{INVESTIGACIÓN Y EXPERIENCIA}

\section{Resumen}

A partir de su experiencia como investigadora, la autora del artículo reflexiona sobre las relaciones entre investigación y experiencia, con el propósito de contribuir a ampliar la posibilidad de reflexión tanto para los investigadores como para aquellos que quieren iniciarse en el campo de la investigación. Según el texto, están dadas las condiciones para deambular entre la investigación y la experiencia, lo cual solo es posible a partir de explicitar qué tiene que ver la una con la otra, así como el lugar del investigador en ese proceso.

Palabras clave: Investigación científica, relación investigación-experiencia

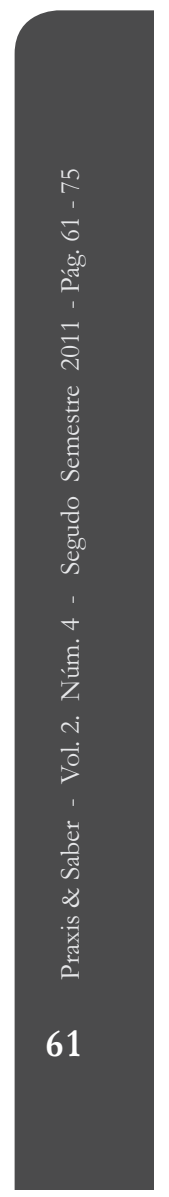




\section{RESEARCH AND EXPERIENCE}

\section{Summary}

Based on personal research experience, the author of the article reflects on the relationship between research and experience, with the intention of helping to extend the reflection to both researchers and those who are starting in the research field. The conditions for moving between research and experience, are given by explaining the relationship between one and the other, as well as the role of the researcher in this process.

Key Words: scientific research, research-experience relationship

\section{RECHERCHE ET EXPÉRIENCE}

\section{Résumé}

A partir de son expérienceen tant que chercheuse, l'auteur de l'article réfléchit sur les relations entre la recherche et l'expérience, ayant comme but la contribution à amplifier la possibilité de réflexion tant pour les chercheurs, tant pour ceux qui veulent s'initier dans le domaine de la recherche. Selon le texte, les conditions pour déambuler entre la recherche et l'expérience sont données, ce qui est seulement possible à partir d'expliciter ce que l'une a à voir avec l'autre, de même que la place du chercheur dans ce processus-là.

Mots clés: Recherche scientifique, relation recherche-expérience.

\section{PESQUisa E EXPERIÊNCIA}

\section{Resumo}

Baseada na sua experiência como pesquisadora, a autora do artigo reflete sobre as relações entre pesquisa e experiência, com o intuito de contribuir para ampliar a possibilidade de reflexão tanto para os pesquisadores como para aqueles que querem se iniciar no plano da pesquisa. Segundo o texto, estão dadas as condições para deambular entre a pesquisa e a experiência, o que é possível só partindo de fazer explícito o quê tem a ver uma com a outra, assim como o lugar do pesquisador nesse processo.

Palavras chave: pesquisa científica, relação pesquisa-experiência 


\section{Introducción}

Las preguntas que quiero presentar son: ¿qué es la investigación y por qué y para qué nos interesa hablar de ella?, ¿que tiene que ver la experiencia con la investigación? y cacaso la investigación se reduce a un campo de prestigio, a un factor diferenciador que crea jerarquías, o es, antes que todo, un lugar de creación, inventiva, desocultamiento y cambio? Estas preguntas han sido planteadas desde dos principios irrenunciables, los cuales permiten mirar la investigación en sus distintas vertientes: en primer lugar, asumirse como un investigador comprometido con la transformación social, con un presente y un horizonte donde coexisten la igualdad y la justicia; en este sentido, la investigación y la política se entrelazan, el compromiso político del investigador implica no sólo su alineamiento irrestricto con la crítica, sino con la acción; asimismo, investigar nace de la pasión del investigador, de su deseo de que las cosas cambien; sin esa pasión, el conocimiento acerca del cómo de la investigación es nada. En segundo lugar, la conciencia de que investigar es involucrarse, dejarse afectar por eso que estamos investigando; consecuentemente, no se investiga un tema, algo externo y contingente respecto del sujeto, sino una situación de vida, una experiencia, que envuelve también al propio investigador y su experiencia; por eso los testimonios nos pesan, los relatos del otro nos hacen mella; desde esa subjetividad afectada, tiene lugar la investigación. Esta manera de vivir la investigación, como algo donde convergen lo público y lo íntimo, genera ciertas suspicacias, recelos y vetos por parte de la academia instituida.

La investigación tradicional, al identificarse con el enfoque neoliberal, al igual que el conjunto de los procesos educativos, se ha transformado en un procedimiento estandarizado, que busca homogeneizar y controlar al sujeto de la experiencia; en este sentido, la investigación ha devenido más y más en una metodología, en herramientas e intentos de simplificar la realidad, cuantificándola, reduciéndola a expresiones genéricas, abstractas y descontextualizadas. La formalización creciente ha legitimado la investigación como parte del dispositivo del cientificismo, como algo superior y diferente a la política y la ideología. Desde los años sesenta, en las ciencias sociales tuvo lugar una naturalización del método hipotético deductivo, incluyendo el análisis de variables, los indicadores, un conjunto de dispositivos, en el marco de un aparato cultural que se autodefinía como ciencia, un conjunto de reglas en un dominio del conocimiento, según Foucault, acompañado de un lenguaje especializado, que unía el empirismo y la teoría. Esta tendencia no se ha detenido, sino que ha llegado, incluso, a permear las formas más alternativas de la investigación. 
Lo que llama la atención hoy es que sigamos apelando a estas viejas formas, así como que estén tan naturalizadas en el campo de la educación. Junto con esta investigación reducida a "metodología de la investigación", ha tenido lugar un interés creciente por la innovación y la calidad educativas, enmarcadas en la búsqueda de la competitividad, la eficiencia y la eficacia, hasta arribar a la equidad; el educador ha devenido no solo un operador de políticas macroestructurales, sino un sujeto convencido de que todo se debe cambiar. El punto es que el cambio está sujeto a reglas predeterminadas, a las áreas que el sistema educativo autoriza.

Camus decía: "conozco algo peor que el odio, el amor abstracto". En nombre del amor abstracto, amor a la libertad, la democracia, la pureza de la raza, los jóvenes..., se han cometido y justificado grandes genocidios. La investigación abstracta pertenece al mismo campo que el amor abstracto. En su nombre se han acumulado las estadísticas despojadas del sujeto, la información que no duele ni compromete, ni lleva a sentirse uno con eso que se está mostrando. En su nombre, se han inventado categorías que despojan al sujeto de su dignidad y de su memoria: los pobres, el rezago educativo, los repitentes, los mutantes, los herederos... Al olvidar el proceso de producción de la realidad, la vida social se deshistoriza y solo se ven los síntomas o los efectos. De este modo, la investigación se pone al servicio del ocultamiento, lo contrario a su sentido originario. Esta manera de hacer investigación, soslayando la experiencia se refleja en varios campos:

a) En el lugar que ocupa la investigación en las carreras de formación de maestros y de profesionales de las ciencias sociales, así como en los posgrados, en particular en las maestrías profesionalizantes; en estos programas, la investigación ha sido reducida a una o más asignaturas, a una línea o espacio curricular, antes que a una experiencia;

b) En la función legitimadora y jerarquizante de la investigación, que lleva a diferenciar los docentes, a secas, de los docentes-investigadores; los prácticos (leáse los maestros), de los investigadores; los semilleros de investigación, de los "tutores". En las diadas anotadas se establecen relaciones jerárquicas. A modo de ejemplo, el tutor, representado por un adulto experto, sostiene el crecimiento de "las semillas", en las cuales se está incubando el saber;

c) En los propios maestros, cuando se busca ser "el maestro-investigador", como un lugar de prestigio, diferenciador de la práctica docente; 
d) En el repudio a la investigación, por parte de algunos maestros, con discursos que afirman que "los maestros no hacemos investigación", sino otra cosa, reflexión desde la práctica, aprendizaje desde la experiencia; en este caso, se plantea la oposición entre investigación y práctica, entre conocimientos de la ciencia y saberes prácticos; en nombre de la experiencia se niega la investigación.

A partir de este análisis inicial entre investigación y experiencia, se desenvuelve el texto.

\section{La investigación como experiencia: primera llamada}

La primera tarea es la desnaturalización de la llamada "investigación", tanto de su enfoque y sus premisas, como de sus prácticas; un ejercicio de volver la pregunta hacia la investigación misma. En vez de investigar algo fuera, en la realidad, la idea es investigar el campo de la investigación, su concepción y su práctica. Asimismo, la propuesta presente en este texto es que la investigación es ante todo una experiencia, algo que nos pasa, nos cambia, nos penetra, nos recrea; así como la lectura es una experiencia, la experiencia de la lectura, donde el lector y el autor se entremezclan. Igualmente, la escritura es, ante todo, la experiencia de la escritura, donde el sujeto es hablado o escrito por la escritura, un sujeto que se deja llevar por la escritura y no el que organiza el texto. Entonces, el sujeto de la investigación puede ser simultáneamente investigador e investigado.

Pero no solo la investigación es una experiencia, sino que parte de la experiencia para construir conocimiento y adoptar nuevamente la experiencia como el lugar al cual se puede llegar, contribuir y fortalecer. Al pensar la investigación como experiencia, el sujeto, individual o colectivo, es ubicado en el centro de esta operación. En este enfoque acerca de la investigación cuenta la experiencia, el sujeto y el sentido de la investigación como experiencia.

\section{Acerca de la experiencia como camino en educación}

Un grupo de filósofos y educadores contemporáneos han visto en la experiencia una nueva manera de acercarse a la educación. Para Larrosa (2003), el par experiencia/sentido, aun cuando no busca garantizar nada, abre nuevos espacios de pensar y hacer, a diferencia del par ciencia/ tecnología o el par teoría/práctica. Según el par ciencia/tecnología, la educación se reduce a ciencia aplicada, cumplimiento de objetivos, 
evaluación de desempeño, estructuras dominadas por expertos; por el contrario, según el par teoría/práctica, la educación es reflexión desde la práctica, práctica política encaminada a la realización de ciertos ideales como la justicia y la igualdad. Larrosa es claro cuando plantea las nuevas posibilidades que brinda la experiencia, concebida como "el tercer camino":

En ese marco, tengo la impresión de que la palabra experiencia o, mejor aún, el par experiencia/sentido, permite pensar la educación desde otro punto de vista, de otra manera. Ni mejor ni peor, de otra manera. Tal vez llamando la atención sobre aspectos que otras palabras no permiten pensar, no permiten decir, no permiten ver. Tal vez configurando otras gramáticas y otros esquemas de pensamiento. Tal vez produciendo otros efectos de verdad y otros efectos de sentido. Y lo que he hecho, o he intentado hacer, con mayor o menor fortuna, es explorar lo que la palabra experiencia nos permite pensar, lo que la palabra experiencia nos permite decir, y lo que la palabra experiencia nos permite hacer en el campo pedagógico. Y para eso, para explorar las posibilidades de un pensamiento de la educación elaborado desde la experiencia, hay que hacer, me parece, dos cosas: reivindicar la experiencia y hacer sonar de otro modo la palabra experiencia (2003: 2).

Al hacer mías las palabras de Larrosa, quiero afirmar que este camino de la experiencia no implica negar el horizonte libertario de la educación, ni el carácter político del hecho educativo, ni la búsqueda de algo nuevo y más justo, sino acercarse a ellos desde otro lugar, justamente "la experiencia", ese lugar que no es la teoría, ni la norma, ni el deber ser.

\section{Qué es la experiencia}

Veamos entonces qué es la experiencia y qué implica "reivindicarla", siguiendo a Larrosa. En primer lugar, dejar de pensar que es algo menor, un saber práctico, contingente. En segundo lugar, quitarle toda autoridad, toda garantía de que si tuve la experiencia, entonces está asegurado un cierto conocimiento, una cierta superioridad. En tercer lugar, dejar de reducirla a un concepto, a algo que se puede "determinar". En cuarto lugar, ubicar la experiencia en la vida social, en el capitalismo salvaje de los últimos tiempos, que con su economicismo pone en crisis la posibilidad de que se diseñen proyectos de vida liberados de la contingencia.

Regresando a Larrosa (2003, 2009a), la experiencia es "lo que nos pasa"; pero el punto es cuándo y cómo nos pasa algo, algo que quiebre la vida 
natural, la rutina, la tradición, el hacerlo sin pensar, porque "así es no más". La experiencia tiene lugar como lo contrario a un sentir que se empieza a hacer habitual en el mundo actual: "no pasa nada", "no me pasa nada" o "no le veo sentido", "no lo puedo manejar". De acuerdo con Melich, la experiencia es un padecer, "es como salir de viaje", "salirse de uno mismo e ir hacia lo otro, hacia el otro" (Melich, 2002, p. 77). La experiencia se presenta como singular, frágil, contingente, no asegurada, sino expuesta, desordenada. El sujeto de la experiencia es un sujeto expuesto, vulnerable, que toma riesgos (Foster, 2009; Larrosa, 2009a). El relato emerge como el vehículo de la experiencia (Foster, 2009).

Finalmente, para Larrosa, "la experiencia es cada vez más rara por falta de tiempo [...]. El sujeto moderno no sólo está informado y opina, sino que es también un consumidor voraz e insaciable de noticias, de novedades. Y la agitación que lo caracteriza también consigue que nada le pase" (2009a, p. 35). Siguiendo a Larrosa, ha tenido lugar un vaciamiento de la experiencia, tanto por el horror (el hombre que regresa de la guerra está mudo, según Benjamin), como por el vértigo de la vida cotidiana (Agamben), el ir de aquí para allá, el sentir que nos movimos todo el día y "no pasó nada", el exceso de trabajo, la hiperactividad, el sentimiento de que todo se puede hacer y cambiar, el deseo siempre de querer lo que no se tiene. "Y por eso, porque siempre estamos queriendo lo que no es, porque estamos siempre activos, porque estamos siempre movilizados, no podemos pararnos. Y al no poder pararnos, nada nos pasa" (Larrosa, 2009a, p. 36). El sujeto que no se detiene a pensarse es el mismo para el cual el trabajo, la escuela o la investigación han dejado de ser lugares de experiencia, para ser lugares de sobrevivencia, de rutina, de tradición, de hacer "lo que hay que hacer", de innovación compulsiva.

\section{¿Qué es esto de la investigación como experiencia?: segunda llamada}

El intento de normalizar la educación ha tenido lugar junto con domesticar la experiencia y la investigación; en vez de experiencia del sujeto, el experimento (o los diseños cuasi experimentales), como un lugar controlado y controlable, limitado a ciertas variables, cada una de las cuales ocupa un lugar predeterminado; en vez de investigación, metodología, análisis de variables, descomposición de un proceso en sus partes, tablas, cuadros, indicadores, formatos, criterios, etapas, informes normalizados... fin del relato. Además, concursos de proyectos de investigación, fondos concursables, investigación sujeta a tiempos y rendimientos, a modas, a temas "que se venden". 
Por su parte, está el investigador, el sujeto que es parte de una institución que construye su identidad, obligado a investigar y publicar, que vive su carrera de investigador como una sucesión de niveles, obstáculos y logros. Él también ha sido privado de su experiencia; investiga lo que es de interés en ese momento para las figuras de poder, lo que tiene financiamiento, lo que da prestigio. Derrida afirmaba que los límites entre lo civil y lo militar se estaban desdibujando, y que toda ciencia era finalista, es decir, "ciencia en última instancia para la guerra". Antes que Derrida, Heidegger, en los cincuenta, hablaba del pensar instrumental y de que nunca se habían escrito tantos informes, $y$, al mismo tiempo, cada vez se pensaba menos... Y entonces la investigación... Cierto, algunos investigadores, algunas instituciones, algunos espacios institucionales, en algunos momentos históricos propicios, buscan y logran quebrar, al menos en parte, estas reglas de juego. Ante este panorama, ¿es posible desmontar la investigación y hacer "otra investigación"? La respuesta es sí, y el camino es la experiencia; una experiencia que es siempre social.

En primer lugar voy a hablar desde mi experiencia..., construida y desarmada en el tiempo, en varios espacios, en una búsqueda que solo fue posible con los otros y en situaciones donde la apertura y la herencia se mezclaron con la ausencia, el desarraigo y la llegada de nuevos sentidos; experiencia, igual que el sujeto que volvía de la guerra, a veces muda, pero viviendo también el vértigo de la hiperactividad y dándome tiempo para detenerme, pensarme e investigar. Sin embargo, "mi experiencia" es la experiencia de muchos, de una generación, de varias generaciones. Un largo proceso que empezó como estudiante de sociología, en la cuna del cientificismo; como estudiante que buscaba otra manera de acercarse a lo social, y no sabía cómo, como parte de un proceso político de masas inédito en la historia de nuestro continente, como parte de una juventud que creía que todo era posible. Escribir hoy sobre la investigación y la experiencia es posible desde esa historia, desde esa generación, desde esa manera de haber sido joven. Haber construido otra forma de hacer investigación sólo se entiende desde ese tiempo político y desde una práctica de investigación que empezó en las formas consagradas y logró ponerlas en cuestión. La práctica cuenta... cuando el sujeto se conmueve con lo que hace, cuando se ve haciendo una práctica que lo reduce a "encuestador" o "entrevistador", a conseguir información, a transformar al otro en un dato, un caso, una frecuencia en un cuadro de doble entrada.

En segundo lugar, comparto algunos aprendizajes en torno a la investigación "desde la experiencia", los cuales, de ninguna manera, 
aspiran a ser un decálogo o un conjunto de lecciones. Los enumero con la finalidad de hacer más claro el texto.

1. Vivir en la pregunta. En palabras de Freire (1986), antes que saber preguntar, el punto es "vivir en la pregunta", estar en una permanente disposición indagativa, es un buscar donde cuenta el proceso, antes que llegar a una verdad única. Cuando la investigación se vive como experiencia, el sentido es desnaturalizar lo establecido; preguntarse si puede ser de otra manera lo que se ve, lo que se analiza, incluso los "resultados" de otros estudios, los propios resultados y las diferentes maneras instituidas de hacer investigación. En la investigación se entrecruza el proceso de construcción de conocimiento con el proceso de desmontar lo que se está haciendo, y lo que ya está hecho. La desestabilización del investigador es una tarea central, que implica navegar en aguas inciertas, poner en cuestión los propios supuestos, relacionar lo que está separado, historizar lo que se presenta como natural, hacer arqueología con marchas y contramarchas, diálogos entre la propia experiencia, la de los otros y la teoría, aceptar todo como provisorio e inconcluso, animarse a revisar, reescribir, volver a empezar, hacer trabajo de campo, regresar a la teoría y, luego, iniciar otra ronda de trabajo de campo, mover los objetivos iniciales, darse cuenta de que la visión inicial era incompleta e incluso falsa. Esta disposición a estar en la pregunta implica también no sentirse obligado por las teorías disponibles, por las teorías de moda, por la comunidad de investigadores, ni tentado por los proyectos más fácilmente aceptados y financiables.

2. Implicación subjetiva y política. Investigar como experiencia es lograr una relación íntima y solidaria con los sujetos que son parte de lo que estoy investigando, acercarme a ellos como iguales, como pares, a los cuales acompaño en su proceso; lo contrario a investigar sobre... o acerca de..., como algo que está fuera de mí, algo que no me incumbe, entonces... me mantengo en mi rol profesional, como un ser aislado. Asimismo, investigar es reconocerse como un sujeto inacabado que quiere aprender, tomar riesgos, transformarse y transformar el mundo..., un sujeto que se reconoce incompleto y dispuesto a aprender con los otros. El que investiga necesita estar abierto al otro, a sí mismo, a los múltiples sentidos, a la transformación permanente. Por su parte, son "los investigados" los que permiten acceder al sentido de lo que se está investigando. Igualmente, investigar es "estar ahî", en el lugar del otro, en el tiempo del otro, en sus 
zapatos, tarea imposible... Un punto importante es reconocer que es imposible, pero se intenta... Consecuentemente, investigar es romper la relación jerárquica de investigador-investigado, experto-ciudadano corriente, investigador versus maestro. Finalmente, investigar es construir con los otros, es un encuentro con eso que se investiga, con esos sujetos, y es un encuentro que se recrea cada día.

3. Compromiso social, político y ético. Siempre se investiga para algo... En este caso, la investigación tiene como norte contribuir no a una política de la guerra, sino a una sociedad más igualitaria; no es para mí, no es para publicar, para el prestigio, es para ellos, para el grupo, a quien me debo, para crear condiciones para su propio proceso de empoderamiento. Investigar es ponerse al servicio del otro. Y la orienta un profundo sentido de justicia, del cual ni siquiera se habla, pero que siempre está allí, acompañado de respeto y amor por toda forma de vida. En consecuencia, un investigador que vive en paz interna y para la paz planetaria.

4. Hacer puentes. Implica transitar de la experiencia a la teoría y viceversa, estar interiorizado del conocimiento disponible en relación con lo que se está investigando, pero al mismo tiempo animarse a buscar y desarrollar otras respuestas; no sentirse constreñido por la academia y por el sentido común de una época. Asimismo, investigar es darse cuenta de la distancia entre el proyecto que escribimos y lo que pasa en la realidad, cuando vamos allá..., y ver cómo las cosas se aclaran entonces... Finalmente, investigar es articular experiencias particulares con teorías, reconociendo las congruencias, pero también las tensiones y las discrepancias; investigar es lo contrario a normalizar los testimonios para que calcen con la teoría. En efecto, investigar es producir conocimiento teórico desde la práctica, desde testimonios particulares, desde historias de vida, que podrían entrar en contradicción con la teoría existente... Investigar, en el mismo sentido, no es "bajar", "aplicar" la teoría a una realidad particular, que entra a modo de "ilustración", ejemplo o evidencia; de este modo, la experiencia, de los otros y la propia, es el centro del proceso de investigación; la teoría coopera, orienta, pero no determina.

5. Revelar las ausencias. Investigar es hacer visibles experiencias que, al no haber sido investigadas, no tenían presencia social. De acuerdo con De Sousa (2006), toda experiencia social que no se comparte y se hace pública, permanece invisible; de allí su propuesta de una sociología de las ausencias. En investigación no sólo es fundamental 
investigar lo no investigado o lo poco investigado, sino desocultar las partes invisibles de temas que han sido investigados una y otra vez o invertir los sentidos que se les han atribuido.

6. Comunidades de aprendizaje. Investigar es crear comunidad entre diferentes, pero unidos por el proyecto común de investigar... para algo. Comunidad es un "circuito de donación recíproca", desde la diferencia (Espósito, 2003). Desde las comunidades se puede realizar una investigación que pone al sujeto en el centro de la acción.

7. El lugar de la escritura. Investigar es escribir, para conservar, pensar y compartir. Investigar es escribir incluyéndose en la escritura, con una posición de reconocimiento de las contradicciones internas y externas; una escritura que no justifica al sujeto que escribe, que no dice lo bien que lo hace, los logros que alcanzó, sino que da cuenta del proceso, en todas sus dimensiones, tensiones, logros, limitaciones. Asimismo, investigar es escribir saliéndose de la lógica del tratado, del texto, que es una trama de afirmaciones deductivamente conectadas, para intentar una escritura fragmentaria, que se construye paso a paso, que transita de una orientación a otra, que presenta una conjetura y se anima a refutarla, que se abre a lo divergente, a las aclaraciones, que sigue la lógica del hipertexto, del texto ramificado, antes que lineal, que elude ser "formateada"... El relato discontinuo, denso, subjetivo, intersubjetivo, donde prima la voz de los otros, la lengua de los otros, interpretada, reinterpretada, junto con la consciencia de ese proceso de alteración de sentido, es la manera de hacer presente una investigación como experiencia.

8. La visión holística y comprensiva. Investigar es mirar lo pequeño, el detalle..., como igual de importante a la grande; asimismo, es estar consciente de que el significante significa, que el significante, el síntoma, es ya "el problema". Finalmente, investigar es eludir la explicación, evitar querer tranquilizarnos con la explicación (esto es así por..., "estas son las causas"...), con ordenar o reordenar la realidad de acuerdo con el "formato" de la investigación.

9. No al sentido impuesto. Escritores de la talla de Kertész, contado por Larrosa (2007), dan cuenta del preguntarse por el sentido de la vida. Kertész se presenta a sí mismo como un sujeto que ha pasado por distintas situaciones (el nazismo, el campo, el estanilismo...), y se pregunta si su vida ha tenido sentido; es el mismo sujeto que en Sin sentido, narrado por Larrosa (2009b), cuenta cómo al regresar del 
campo de concentración es "interrogado" por un periodista, que le demanda que cuente su historia, que le hable de las miserias del campo; Kertész, a pesar de su juventud, se niega a hacerlo, se da cuenta de que eso sería perder lo único que tiene, su experiencia, para que el otro la transforme en historia pública, en memorial, en noticia; su respuesta es que lo que recuerda del campo es el aburrimiento, tener que ocupar el tiempo, la sobrevivencia, la adaptación a lo que había, como toda la vida. Kertész se niega a que alguien desde afuera atribuya sentidos a su experiencia; prefiere dejarla como es, incomprensible, absurda.

10. La investigación-experiencia como disposición de vida. En vez de concebir la investigación como un espacio curricular en los diferentes niveles educativos, o como una actividad profesional y especializada (hacer proyectos, hacer estudios), necesitamos pensarla como una disposición de vida, continua, que no se limita a unas horas al día, a un trabajo de investigación; como una disposición a mirar la realidad, aprender de ella y ganar en experiencia.

11. La investigación-experiencia como fundamento curricular. Si aceptamos la idea de que la escuela priva al sujeto de la posibilidad de la experiencia, está pendiente la tarea de lograr lo contrario. En consecuencia, la investigación concebida como experiencia puede contribuir a este proceso de inversión institucional, haciéndose un fundamento del currículo, no un nicho especializado, en todos los niveles educativos, desde la educación inicial hasta la universidad. En el caso particular de los posgrados, se sugiere observar la proliferación de maestrías de carácter profesionalizante, que buscan hacer del estudiante un experto en algún campo de la educación (la gestión, la evaluación, la investigación, el aprendizaje). Por el contrario, la propuesta de este texto es crear condiciones para que los estudiantes de maestría vivan la investigación como experiencia y se fortalezcan como sujetos.

12. El "maestro investigador". Ante la estigmatización del maestro como práctico y de naturaleza "inferior" respecto de los investigadores, se propone promover la emergencia del maestro investigador; sin embargo, esta opción implica impulsar en los maestros la disposición a "vivir en la pregunta", antes que a ser un ejecutor de proyectos de investigación.

13. La investigación es de todos. Se están desarrollando en América Latina las redes de maestros; junto con ellas, una corriente de 
pensamiento que, nacida de las estigmatizaciones que han sufrido los maestros, así como de las presiones para convertirlos en "maestros investigadores" en un sentido profesionalizante, afirma que no es tarea de los maestros hacer investigación, sino reflexión desde la práctica; posición que deja a los maestros fuera del campo de la investigación. Por el contrario, la postura que se suscribe en este texto es que los maestros, al mirar su propia práctica, pueden estar haciendo investigación, si lo hacen con esta disposición hacia la crítica, la solidaridad y la apertura.

Hago mías estas palabras de Contreras y Pérez de Lara: "esto que estamos diciendo no tiene que ver sólo con una manera de entender la investigación, sino que es también consustancial con una manera de entender la educación" (2010, p. 70). En uno y otro caso, el saber de la experiencia se nutre del otro, de la relación con el otro en que yo mismo me transformo. La investigación de la experiencia no se limita a mostrar tendencias, ni a dar cuenta, ni siquiera a desocultar, sino implica fluir con la realidad, permitiendo que eso que estamos investigando nos haga otros. Recuerdo cómo sentí la diferencia entre lo que suponía que sabía y lo que quería investigar, y el encuentro con la palabra del otro, en ese caso, de un joven egresado de la secundaria técnica; en un rato, todo mi saber previo se había trastocado; de mi parte, tuvo lugar el reconocimiento de la distancia entre la teoría y el sujeto, y la sensación de apertura que hace posible la palabra del otro.

En suma, la propuesta es un investigador que elude las dicotomías, la separación entre teoría y práctica, entre subjetividad y objetividad, entre realidad y sujeto, entre investigación y escritura, entre ciencia y política. Un investigador abierto a la incertidumbre, que viaja de un espacio a otro, tanto en términos simbólicos como físicos, un sujeto dispuesto a construir y desmontar al mismo tiempo. Un investigador que es sujeto de la experiencia y al mismo tiempo es uno con los otros y con un proyecto de vida, una forma de hacer comunidad, donde la aspiración de la igualdad en la diferencia está siempre presente.

\section{Epílogo}

La responsabilidad del investigador no se limita a investigar algo; tampoco a investigar junto con, o a acompañar procesos sociales y educativos de empoderamiento desde la sociedad civil. Las responsabilidades del investigador son múltiples; por un lado, compartir lo que sabe y lo que es, compartirse en espacios de reflexión/formación de diferente tipo y grado de formalización; por otro, es tarea del investigador preguntarse por su 
relación con las políticas públicas. En los últimos años se ha incrementado el interés de los investigadores por "la incidencia" de la investigación en las políticas; al mismo tiempo, otros investigadores han planteado la imposibilidad del diálogo entre el investigador y el político. En este marco, necesitamos preguntarnos cómo pueden los investigadores organizados en comunidades de aprendizaje integrar acción y reflexión, sin por ello hacerse cómplices de los tomadores de decisiones a nivel gubernamental.

Otra tarea igualmente relevante que toca a los investigadores es desmontar la lógica de los enfoques y políticas predominantes en el campo de la educación; la educación por competencias y las innovaciones son dos espacios donde los investigadores tienen mucho que decir. Ante el enfoque de competencias es responsabilidad del investigador mostrar las consecuencias de esta perspectiva, desde la fragmentación curricular hasta el olvido del sujeto; esta posición es contraria a decir que, dado que las competencias están en el campo de la educación, no queda otra alternativa que involucrarse con ellas. En relación con las innovaciones, el investigador necesita dar cuenta de cómo desde los noventa las innovaciones han seguido una lógica de centro, donde perviven mecanismos de replicación y generalización, que llevan a subordinar los saberes locales. En el mismo sentido, el investigador puede contribuir a sistematizar y fortalecer experiencias significativas que se generan desde sus propios contextos, donde los sujetos son el eje, antes que las estructuras.

En este marco, es tarea del investigador contribuir a la diferenciación entre seudoinnovaciones e innovaciones que se alejan de la lógica oficial; así como entre educación básica o educación "mínima", entre calidad de los aprendizajes o subordinación de la enseñanza a la evaluación. También es tarea del investigador contribuir a una pedagogía del sujeto o de la experiencia, que se nutra de las ciencias sociales y la filosofía, sin por ello perder su singularidad. En este plano, el investigador necesita dar visibilidad a los temas ausentes en el discurso pedagógico, como la relación entre sujeción y formación, o la degradación educativa creciente, que pone de manifiesto la presencia de la política del control, tanto en los dispositivos pedagógicos como didácticos (Gómez Villalpando, 2010).

Finalmente, el investigador necesita pensar en el sentido de la investigación, arriesgándose a transitar caminos poco frecuentes, que implican siempre el riesgo del rechazo por parte de los pares académicos (Granja, citada por Villalpando, 2010).

Cuando decimos investigador, decimos comunidad. 


\section{Referencias}

Contreras, José y Pérez de Lara, Nuria (2010). Investigar la experiencia educativa. Madrid: Morata.

De Sousa Santos, Boaventura (2006). "La sociología de las ausencias y la sociología de las emergencias: para una ecología de saberes". En: Renovar la teoría crítica y reinventar la emancipación social, Capítulo I, CLACSO. Disponible en: www.clacso.org.ar/biblioteca)

Espósito, Roberto (2003). Communitas. Origen y destino de la comunidad. Buenos Aires: Amorrortu.

Freire, Paulo (1986). Hacia una pedagogía de la pregunta. Buenos Aires: La Aurora.

Foster, Ricardo (2009). "Los rostros de la alteridad". En: Skliar, Carlos y Larrosa, Jorge (comp.): Experiencia y alteridad en educación. Buenos Aires: Homo Sapiens.

Gómez Villalpando, Armando (2010). La formación y la sujeción en el discurso teórico de la educación en México. México: Plaza y Valdés.

Larrosa, Jorge (2003). La experiencia y sus lenguajes. (Conferencia). Disponible en: www.me.gov.ar/curriform/publica/oei_20031128/ ponencia_larrosa.pdf. (Consultado en marzo del 2007).

Larrosa, Jorge (2009a). "Experiencia y alteridad en educación". En: Skliar, Carlos y Larrosa, Jorge (comp.): Experiencia y alteridad en educación. Buenos Aires: Homo Sapiens.

Larrosa, Jorge (2009b). "Veinte minutos en la fila. Sobre experiencia, relato y subjetividad en Imre Kertész". Actualidades Pedagógicas Número 54, Revista de la Facultad de Ciencias de la Educación, Universidad de La Salle, Bogotá, julio-diciembre 2009.

Melich, Carlos (2002): Filosofía de la finitud. Barcelona: Herder. 